\title{
Anti-proliferation and anti-metastasis effect of barbaloin in non-small cell lung cancer via inactivating p38MAPK/Cdc25B/Hsp27 pathway
}

\author{
ZHANG ZHANG $^{1}$, WEI RUI ${ }^{2}$, ZI-CHEN WANG ${ }^{1}$, DA-XIN LIU ${ }^{3}$ and LIN DU ${ }^{1}$ \\ Departments of ${ }^{1}$ Acupuncture and Moxibustion, ${ }^{2}$ Supply, and ${ }^{3}$ Traditional Chinese Medicine, \\ Dongzhimen Hospital of Beijing University of Chinese Medicine, Beijing 100700, P.R. China
}

Received January 9, 2017; Accepted June 7, 2017

DOI: $10.3892 / o r .2017 .5760$

\begin{abstract}
Non-small cell lung carcinoma (NSCLC) is the most common lung cancer with high morbidity and mortality. The traditional treatment for NSCLC is particularly liable to relapse with many side-effects. Barbaloin is a natural compound with anticancer efficacy. The present study aimed to investigate the anticancer potential of barbaloin in NSCLC. The results displayed that barbaloin inhibited the viability of A549 cells by decreasing cell growth and the expression level of Ki-67 and proliferating cell nuclear antigen (PCNA), especially at high concentrations (50 and $100 \mu \mathrm{M})$. Besides, barbaloin increased the apoptosis rate of A549 cells and induced an accumulation of G2/M phase. Increased expression of apoptosis-related proteins (caspase-3, -8 and -9) and the changed levels of cell cycle checkpoint proteins (p27, p53 and cyclin A) further convinced of the anti-viability effect of barbaloin in A549 cells. On the other hand, barbaloin significantly suppressed the invasion and migration of A549 cells, and restrained the expression of tumor metastasis-related proteins. We further explored the activation of pro-survival or prometastasis signaling pathways, including AKT, nuclear factor kappa B (NF- $\mathrm{B})$, mitogen-actived protein kinase (MAPK) and $\beta$-catenin. The results revealed that barbaloin inactivated the p38MAPK/Cdc25B/Hsp27 pathway by inhibiting p38 nucleus translocation, while no significant influence was
\end{abstract}

Correspondence to: Dr Lin Du, Department of Acupuncture and Moxibustion, Dongzhimen Hospital of Beijing University of Chinese Medicine, No. 5 Hai Yun Cang Road, Dongcheng, Beijing 100700, P.R. China

E-mail: dulindzm@163.com

Abbreviations: NSCLC, non-small cell lung cancer; PCNA, proliferating cell nuclear antigen; MMP, matrix metalloproteinase; VEGF, vascular endothelial growth factor; MAPK, mitogen-actived protein kinase

Key words: barbaloin, non-small cell lung carcinoma, p38 mitogenactived protein kinase, proliferation, metastasis observed among other pathways. Finally, barbaloin restrained the growth and hepatic metastases of A549 cells in vivo. Taken together, our research indicated that barbaloin inhibited the proliferation and metastasis of NSCLC cells in vivo and in vitro. This may provide safer and more effective aspects for the treatment of NSCLC.

\section{Introduction}

Lung cancer is by far the biggest cancer killer in the world. Almost a million people die from lung cancer every year worldwide, and nearly 600,000 in China (1). Though many advances have been achieved in clinical and experimental oncology, lung cancer treatment is still not satisfactory, with a 5-year overall survival rate of barely $11 \%$ (2). Non-small cell lung carcinoma (NSCLC) has the highest incidence in lung cancers, accounting for approximately $85 \%$ of lung neoplasms $(3,4)$. Research into NSCLC is restricted, due to the complex cell proliferation mechanism and delayed diagnosis (5). In general, chemotherapy combined with surgical resection is the classic therapeutic strategy for NSCLC (6). However, chemotherapy always comes with some critical side-effects, such as leukopenia, hepatic dysfunction and disorder in renal function $(7,8)$. Hence, more secure therapeutic strategies and more thorough studies are needed to improve therapeutic actuality of NSCLC.

Barbaloin, also named as aloin $\left(\mathrm{C}_{21} \mathrm{H}_{22} \mathrm{O}_{9}\right)$, is a natural bioactive anthracycline. Barbaloin is usually extracted from Aloe barnadensis Miller leaves (9). Considerable number of reports have shown the anti-inflammatory, antimicrobial, antioxidant activities, anti-virus and anticancer potentials of barbaloin (9). Besides, previous studies identified the effective role of barbaloin in regulating cell cycle arrest and apoptosis in human cancers, such as colon (10), breast (11), ovarian cancer (12), uterine carcinoma (13). Aloe emodin has been demonstrated to suppress the invasion and metastasis of breast cancer MDA-MB-231 cells (14) and human ovarian cancer cell line HO-8910PM (15). These investigations illustrated the potential of barbaloin in suppressing the proliferation and metastasis of cancers.

In addition, other researchers suggested that aloe-emodin inhibited the invasion of nasopharyngeal carcinoma cells by downregulating the expression of MMP-2 via p38 mitogen- 
actived protein kinase (p38MAPK)-NF- $\kappa \mathrm{B}$-dependent pathway (16). Some others pointed out that p38 was an important determinant of apoptotic death induced by aloe-emodin in NSCLC H460 cells (17). However, no study has indicated the anticancer potential of barbaloin in NSCLC.

In the present study, we investigated the possible anticancer effects of aloin on NSCLC in vitro and in vivo and the underlying molecular mechanisms. The results demonstrated that barbaloin inhibited the proliferation of A549 cells, promoted cell apoptosis and induced a G2/M cell cycle arrest. Further research identified that barbaloin suppressed the invasion and migration ability of A549 cells. The in vivo experiment convinced that barbaloin restrained the growth and hepatic metastases of A549 cells in nude mice, and involved inactivation of the p38MAPK signaling pathway. Our research may present new aspects in the treatment of NSCLC.

\section{Materials and methods}

Animal ethics. NOD/SCID mice were euthanized via the abdominal injection a lethal dose of pentobarbital sodium. All of the animal work was conducted according to relevant national and international guidelines and was approved by the Animal Experimental Ethics Committee of The First Affiliated Hospital of Xinxiang Medical University.

Cell lines and cell cultures. Human NSCLC cell line A549 was obtained from the American Type Culture Collection (ATCC; Manassas, VA, USA), and cells were pre-covered with Matrigel (BD Biosciences, Shanghai, China) at $5 \mu \mathrm{g} /$ $\mathrm{cm}^{2}$. Then, cells were cultivated in modified Eagle's medium (MEM; Invitrogen, Carlsbad, CA, USA), supplemented with $20 \%$ fetal bovine serum (FBS; Invitrogen) and $1 \%$ antibioticantimycotic (Invitrogen). The cells were cultured according to the supplier's instruction, at $37^{\circ} \mathrm{C}, 5 \% \mathrm{CO}_{2}$.

Cell viability analysis. Barbaloin (HPLC $\geq 98 \%$ ) was purchased from Sigma-Aldrich (St. Louis, MO, USA). Barbaloin was dissolved in dimethyl sulfoxide (DMSO) at concentration of $20 \mathrm{mmol} / 1$ and stored at $-20^{\circ} \mathrm{C}$. To evaluate cell viability, $2 \times 10^{3}$ A549 cells were seeded onto 96-well plates coated with $0.1 \%$ gelatin and allowed to attach overnight. Human NSCLC cell lines A549 were directly exposed to various concentrations of barbaloin $(0,10,50$ and $100 \mu \mathrm{M})$ for $72 \mathrm{~h}$. Cell viability was identified using methylthiazol tetrazolium (MTT) assay. Briefly, $20 \mu \mathrm{l}$ of MTT dye solution $(5 \mathrm{mg} / \mathrm{ml}$; Sigma-Aldrich) was added to each well and incubated for $4 \mathrm{~h}$. Then, the old medium was discarded and fresh medium containing MTT (5 mg/ml MTT in PBS; Sangon Biotech, Co. Ltd., Shanghai, China) was added and incubated further for $4 \mathrm{~h}$. Then, DMSO was used to dissolve the formazan. Finally, the OD was determined with a microplate spectrophotometer (ELx800; BioTek Instruments Inc. Winooski, VT, USA) at a wavelength of $470 \mathrm{~nm}$. Experiments were carried out in triplicate.

Western blotting. The expression of key proteins involved in the biological functions of NSCLC cells was measured by western blotting. Cells were treated with different concentrations of barbaloin $(0,10,50$ and $100 \mu \mathrm{M})$. Total cell lysate prepara- tion and western blot analysis were performed according to a previous report (18). Briefly, equal amounts of protein $(40 \mu \mathrm{g})$ were resolved on (6-12\%) SDS-PAGE, electrotransferred onto PVDF membranes (Millipore, Billerica, MA, USA), probed with specific antibodies. The following primary antibodies were used: anti-Ki-67, anti-proliferating cell nuclear antigen (PCNA); anti-caspase-3; anti-caspase-8; anti-caspase-9; antip27; anti-p53; anti-cyclin A; anti-matrix metalloproteinase (MMP)-2; anti-MMP-9; anti-MMP-14; anti-vascular endothelial growth factor (VEGF); anti-AKT; anti-nuclear factor kappa $\mathrm{B}(\mathrm{NF}-\kappa \mathrm{B})$; anti-p38 mitogen-actived protein kinase (p38MAPK); anti- $\beta$-catenin; anti-p-AKT; anti-p-NF- $\mathrm{B}$; anti-p-p38MAPK; anti-p- $\beta$-catenin and anti-GAPDH (Santa Cruz Biotechnology, Santa Cruz, CA, USA), which was used as the internal reference. After incubation with the appropriate horseradish peroxidase (HRP)-conjugated secondary antibody, proteins were detected using a ChemiDoc XRS imaging system and Quantity One analysis software (Bio-Rad Laboratories, San Francisco, CA, USA).

Cell apoptosis analysis. Cell apoptosis of A549 cells was detected by the Annexin V apoptosis detection kit (Beyotime Institute of Biotechnology, Shanghai, China) following a previous study (19). Briefly, the medium containing the floating cells was collected, and the attached cells were washed and trypsinized. The floating and trypsinized cells were collected in the same centrifuge tubes. After centrifugation and washing, the cell pellets were fixed in cold $70 \%$ ethanol. The fixed cells were stained with propidium iodide (PI) and PE-Annexin V. Cell apoptosis percentage was reflected by Annexin V/PI ratio, detected by a flow cytometer (BD Biosciences, Shanghai, China).

Cell cycle analysis. The analysis was measured by flow cytometry (BD Biosciences, San Jose, CA, USA). In short, A549 cells at $1 \times 10^{6}$ cells/well were treated with different concentrations of barbaloin. The cells were then harvested and fixed in $70 \%$ ethanol overnight at $4^{\circ} \mathrm{C}$. Cell nuclei were stained with PI for $30 \mathrm{~min}$. A total of $10^{4}$ nuclei were examined in a FACSCalibur flow cytometer (Becton-Dickinson, Mountain View, CA, USA) and DNA histograms were analyzed by CellQuest software (Becton-Dickinson). Results are presented as the percentage of cells in each phase.

Wound healing assay. The migratory activity of A549 cells was assessed using the scratch assay according to standard methods. A narrow area on the confluent A549 monolayers in 6-well plates were scratched off with a p200-pipette tip. After washing, cells were treated with indicated concentrations of barbaloin. Image acquisition of wound fields was done after removal of inserts $(0 \mathrm{~h})$ and wound closure documentation was done after $24 \mathrm{~h}$ with a phase-contrast microscope (Leica DM IL; Leica Microsystems, Wetzlar, Germany) equipped with a digital camera (Leica DFC300 FX). Images were taken from the same areas as those recorded at zero time and the numbers of the migrated cells were counted.

Transwell invasion assay. The in vitro cell invasion assay was performed in the 24-well plates by a modified Boyden chamber coated with Matrigel (Becton-Dickinson, Franklin Lakes, NJ, 


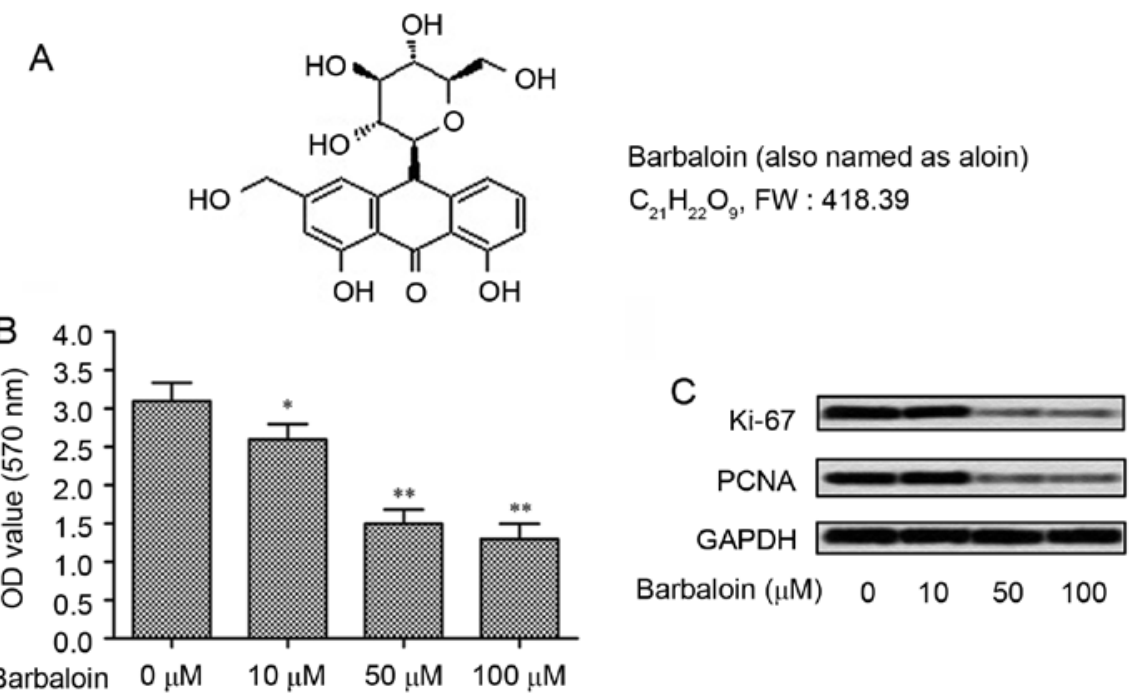

Figure 1. Barbaloin inhibits the growth of NSCLC cells. A549 cells were treated with barbaloin at different concentrations $(0,10,50$ and $100 \mu \mathrm{M})$. (A) The structure of Aloin. (B) The proliferation of A549 cells was determined by MTT assays. Experiments were repeated at least 3 times with similar results, and error bars represent \pm SD. Cells treated with $0 \mu \mathrm{M}$ barbaloin was used as the control group. ${ }^{*} \mathrm{P}<0.05,{ }^{* *} \mathrm{P}<0.01$ vs. control group. (C) The expression of proliferation markers (Ki-67 and PCNA) was meausred by western blot analysis in A549 cells. GAPDH was used as an endogenous reference.

USA). A single-cell suspension containing $2 \times 10^{4}$ A549 cells were treated with barbaloin $(0,10,50$ and $100 \mu \mathrm{M})$ and loaded into the upper chamber, whereas $20 \%$ FBS was added to the medium in the lower chamber. After incubating for $24 \mathrm{~h}$, noninvading cells were removed from the top well with a cotton swab, while the bottom cells were fixed in $95 \%$ ethanol, and stained with hematoxylin. Invasiveness was determined by counting the cells that migrated through the filter.

Immunofluorescence staining. Fluorescent cells were cultured on an 8-well chamber CultureSlides (Becton-Dickinson, Bedford, MA, USA). After $8 \mathrm{~h}$, cells were fixed in 3\% paraformaldehyde in phosphate-buffered saline (PBS) at room temperature for $8 \mathrm{~min}$, then permeabilized with $0.2 \%$ Triton $\mathrm{X}-100$ for $15 \mathrm{~min}$ at room temperature. After washing in PBS, the cells were incubated with primary mouse anti-p38 monoclonal antibody $(1 \mathrm{mg} / \mathrm{ml}$; Transduction Laboratories, Lexington, KY, USA) at $4^{\circ} \mathrm{C}$ overnight. After washing, cells were incubated with biotinylated goat anti-mouse IgG (Pierce, Rockford, IL, USA) at room temperature for $1 \mathrm{~h}$. The immunoreactivity was revealed using Alexa568-conjugated streptavidin (Molecular Probes, Eugene, OR, USA), and cells were counterstained with $10 \mathrm{mg} / \mathrm{ml}$ DAPI. The cells were examined under a Nikon fluorescence microscope (Image Systems, Columbia, MD, USA).

Subcutaneous NSCLC xenografts. All the animals involved in this study were purchased from the the Institute of Zoology, Chinese Academy of Medical Sciences (NOD/SCID mice, clean, 8-weeks old and weighing 20-22 g). A549 cells were digested by the pancreatic enzymes and the final concentration was adjusted to $1 \times 10^{6} / \mathrm{ml}$. On day 0 , the mice $(\mathrm{n}=50)$ were administered with 2001 of $0.75 \%$ sodium pentobarbital solution per mouse and then subcutaneous injection was conducted of $5 \times 10^{6}$ A549 cells. The mice in treatment group $(n=25)$ were injected with barbaloin $(40 \mathrm{mg} / \mathrm{kg}$ body weight) every other day, while the mice $(n=25)$ in model group received the same volume injection of saline. After development of a palpable tumor, the tumor volume was monitored every 5 days, briefly, tumor was isolated from five mice in each group, the volume was assessed by the formula: tumor volume $\left(\mathrm{mm}^{3}\right)=$ maximal length $(\mathrm{mm}) \mathrm{x}$ perpendicular width $(\mathrm{mm})^{2} / 2$. All mice were assigned to euthanasia at the end of the measurements. All animal experiments were performed according to current guidelines and under a protocol approved by the Institutional Animal Care and Use Committee.

Ex vivo fluorescence imaging of the liver. Fluorescence in livers from NSCLC xenograft mice was observed using the Xenogen IVIS spectrum imager (Caliper Life Sciences, Inc., Hopkinton, MA, USA). The total signal intensities were quantified by drawing the region of interest (ROI) using the matching analysis software package supplied by the manufacturer.

Statistical analysis. All results are presented as mean \pm SD. The statistical significance of the studies was analyzed using Student's t-test. The difference was considered statistically significant at $\mathrm{P}<0.05$.

\section{Results}

Barbaloin inhibits the growth of NSCLC cells. To explore the effect of barbaloin on cell viability of NSCLC cells, the NSCLC A549 cells were treated with barbaloin at different concentrations $(0,10,50$ and $100 \mu \mathrm{M})$. The structure of barbaloin is shown in Fig. 1A. The anti-growth effect of barbaloin was measured even at low concentrations $(10 \mu \mathrm{M})(\mathrm{P}<0.05$; Fig. 1B), while higher concentrations (50 and $100 \mu \mathrm{M})$ of barbaloin strongly decreased the OD values of A549 cells $(\mathrm{P}<0.001)$. To confirm the results, the expression of proliferation markers (Ki-67 and PCNA) were measured by western blot analysis. Results displayed that barbaloin reduced the level of Ki-67 and PCNA, especially at higher concentrations 

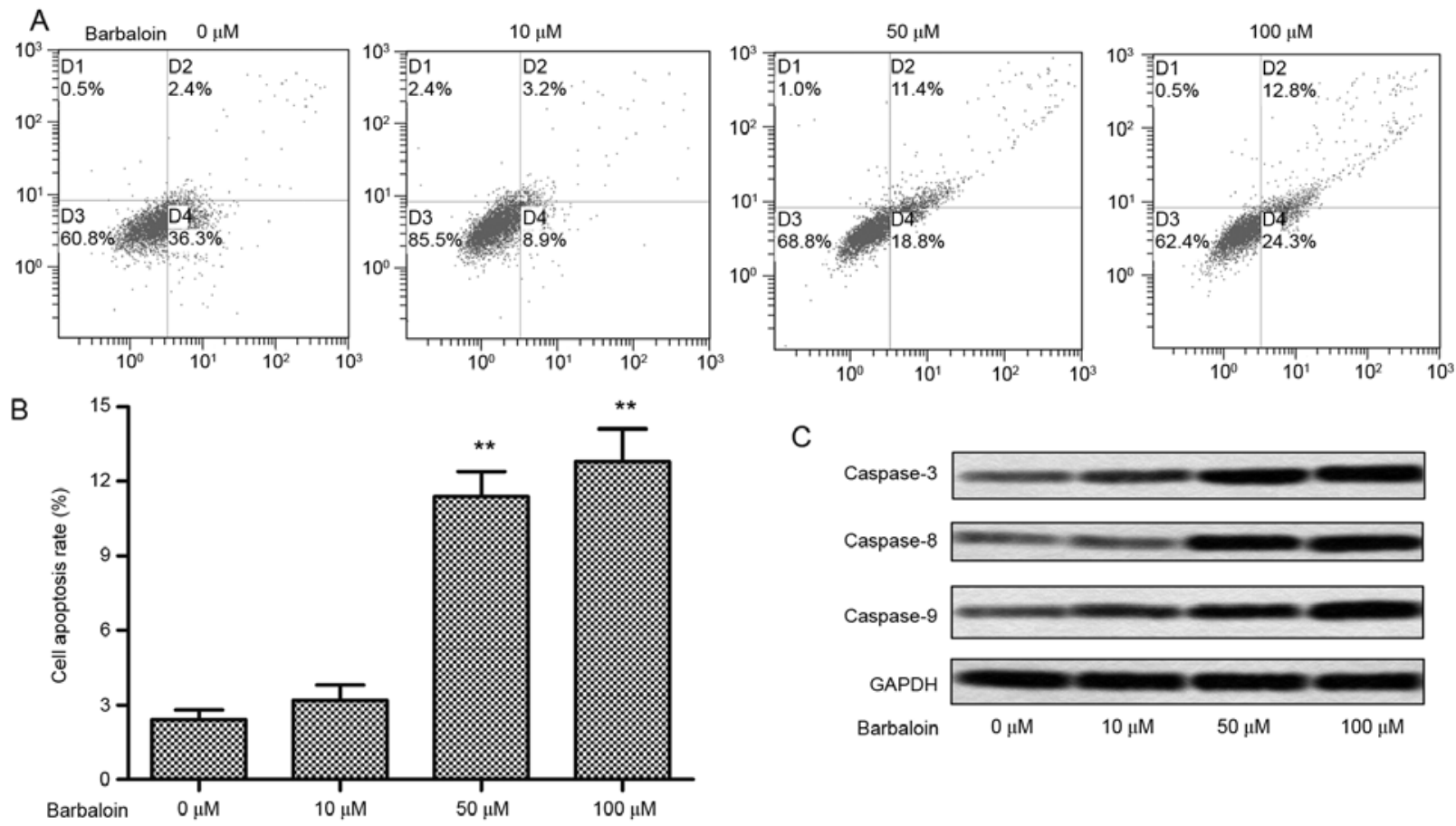

C
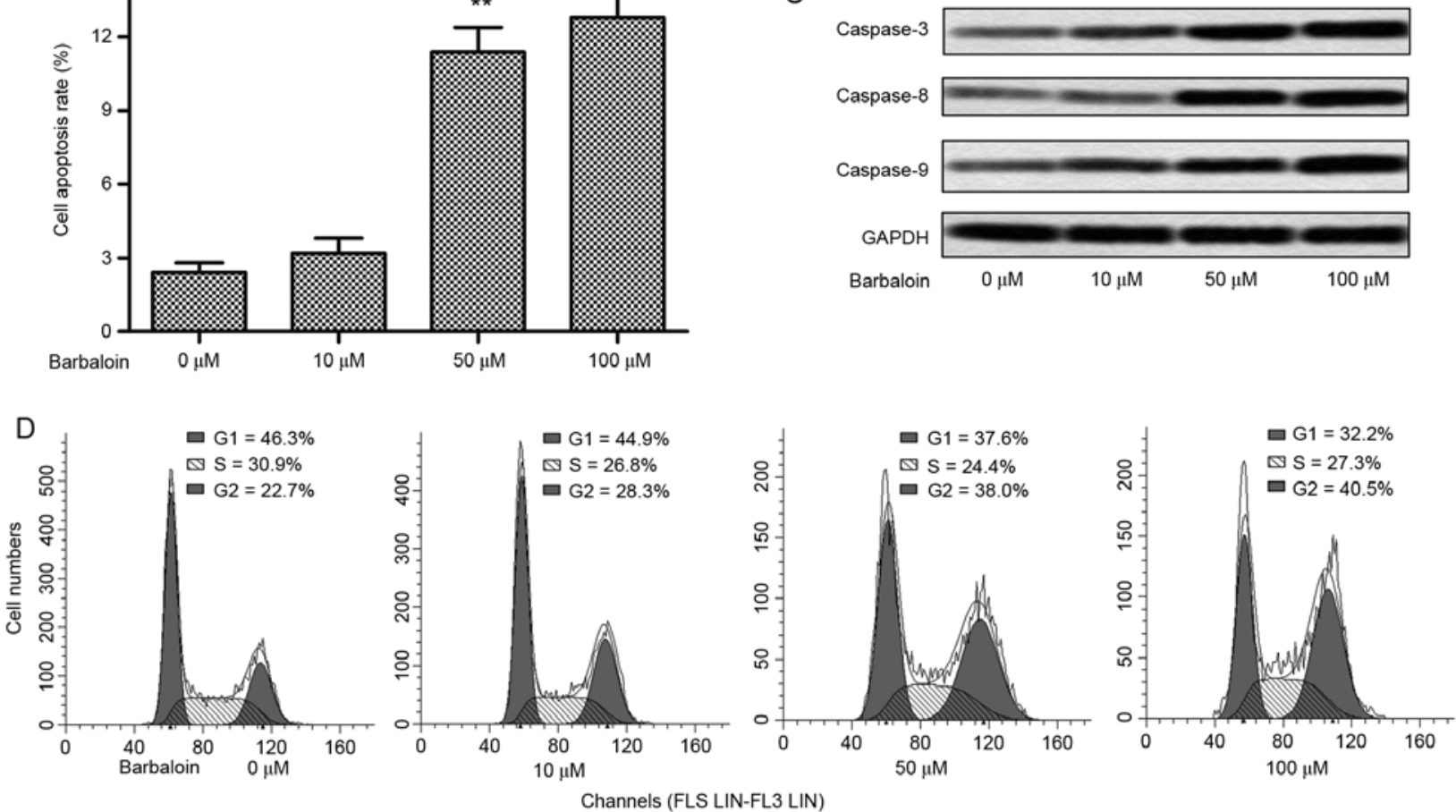

Channels (FLS LIN-FL3 LIN)
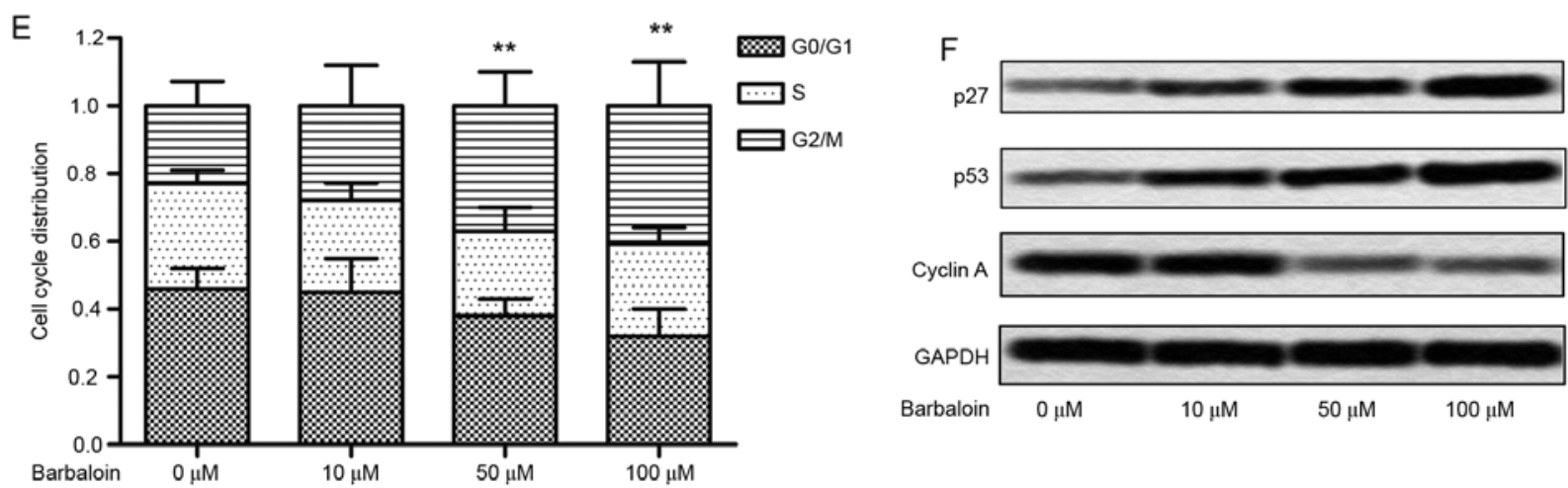

Figure 2. Barbaloin induces apoptosis and G2/M cell cycle arrest in A549 cells. A549 cells were treated with barbaloin at different concentrations $(0,10,50$ and $100 \mu \mathrm{M}$ ). (A) Flow cytometry was used to detect the apoptotic cells in barbaloin treated A549 cells. (B) Data summary and analysis of the cell apoptosis rate in A549 cells according to the results of flow cytometric analysis $\left({ }^{* *} \mathrm{P}<0.01\right.$ vs. control group). (C) The expression of apoptosis-related proteins (caspase- $3,-8$ and -9) in related cells was evaluated through western blotting. GAPDH was used as an endogenous reference. (D) Flow cytometry was performed to determine the percentages of cell population in different phases of cell cycle in related A549 cells. (E) Histogram represents the statistical analysis of cell cycle distribution ( ${ }^{* *} \mathrm{P}<0.01$ vs. control group). (F) The production level of cycle checkpoint proteins (p27/p53/cyclin A) was detected through western blotting. GAPDH was used as an endogenous reference.

(50 and $100 \mu \mathrm{M})$ (Fig. 1C). These results indicated that barbaloin could effectively inhibit the viability of NSCLC cells.

Barbaloin induces apoptosis and G2/M cell cycle arrest in A549 cells. To further investigate the impact of barbaloin on the viability of NSCLC cells, we studied whether barbaloin was capable of affecting apoptosis and the cell cycle. Flow cytometric analysis indicated that no significant difference of apoptosis was detected in A549 cells treated with $10 \mu \mathrm{M}$ barbaloin, whereas 50 and $100 \mu \mathrm{M}$ barbaloin strongly induced cell 
A

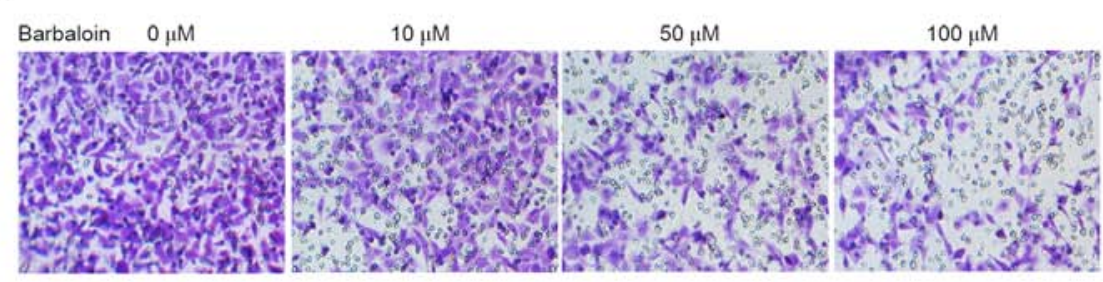

C
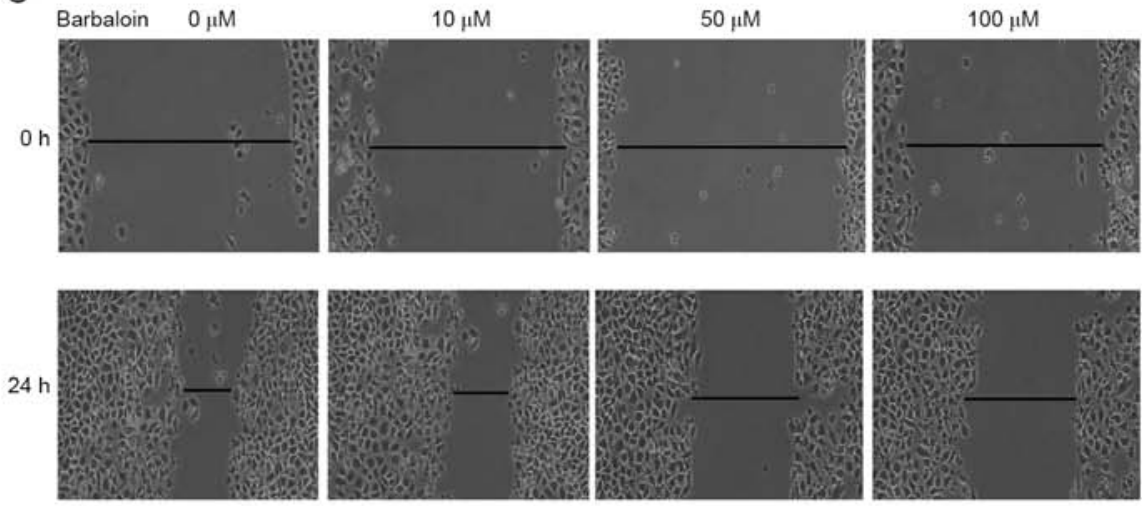
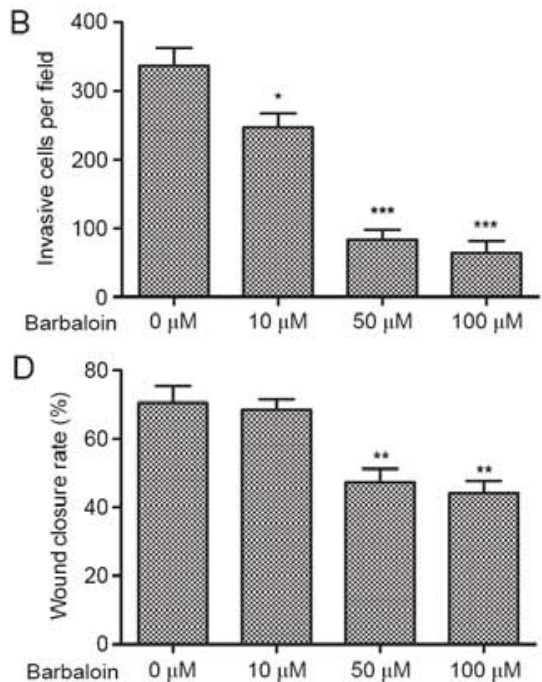

E

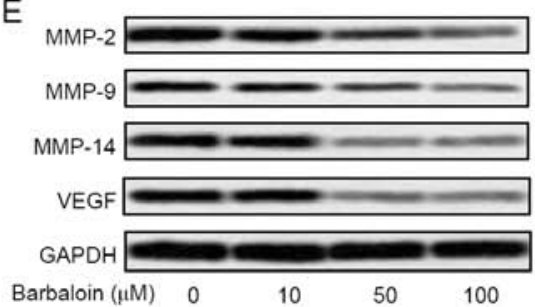

Figure 3. Barbaloin suppresses invasion and migration of A549 cells. A549 cells were treated with barbaloin at different concentrations $(0,10,50$ and $100 \mu \mathrm{M})$ (A) Transwell invasion assays were conducted to observe the invasive A549 cells. (B) Histogram represents the statistical analysis of wound-healing assay ( $\mathrm{P}<0.05,{ }^{* * *} \mathrm{P}<0.001$ vs. control group). (C) The migration rate of A549 cells treated with barbaloin for $24 \mathrm{~h}$ was observed through wound-healing assays. (D) Histogram represents the statistical analysis of wound-healing assays ( ${ }^{* *} \mathrm{P}<0.01$ vs. control group). (E) The expression of tumor metastasis-related proteins (MMP-2/MMP-9/MMP-14/VEGF) was detected through western blotting. GAPDH was used as an endogenous reference.

apoptosis ( $\mathrm{P}<0.01$; Fig. 2A and B). Similarly, the expression of apoptosis-related proteins (caspase- $3,-8$ and -9 ) was enhanced under treatment of barbaloin, especially at higher concentrations (50 and $100 \mu \mathrm{M})$ (Fig. 2C). The analysis of cell cycle indicated that an accumulation of $\mathrm{G} 2 / \mathrm{M}$ phase was caused by $10 \mu \mathrm{M}$ barbaloin $(\mathrm{P}<0.05)$, and the $\mathrm{G} 2 / \mathrm{M}$ cycle arrest was strongly induced by 50 and $100 \mu \mathrm{M}$ barbaloin $(\mathrm{P}<0.01$; Fig. 2D and E). The elevated level of cell cycle checkpoint proteins (p27 and p53) and decreased level of cyclin A further convinced of the cycle arrest induced by barbaloin (Fig. 2F) These results indicated that barbaloin induced cell apoptosis and cell arrest in NSCLC.

Barbaloin suppresses the invasion and migration of A549 cells. The former results revealed the anti-viability effect of barbaloin, we next investigate the effect of barbaloin on the motility of NSCLC cells (A549) by Transwell assays and scratch assays. The number of invasive cells was decreased over 3-fold adding barbaloin (50 and $100 \mu \mathrm{M}$ ) in A549 cells $(\mathrm{P}<0.001$; Fig. $3 \mathrm{~A}$ and $\mathrm{B})$. The results of scratch assays were in agreement with the Transwell assays. The A549 group showed a large closure of the gap, whereas barbaloin (50 and $100 \mu \mathrm{M})$ increased the gap by $\sim 30 \%(\mathrm{P}<0.01$; Fig. $3 \mathrm{C}$ and $\mathrm{D})$. Further western blot analysis indicated that the levels of tumor metastasis-related proteins (MMP-2, MMP-9, MMP-14 and VEGF) were decreased by barbaloin at different concentrations (Fig. 3E). These results suggested the inhibition effect of barbaloin on the motility of NSCLC.
Barbaloin inactivates the $p 38 M A P K / C d c 25 B / H s p 27$ pathway. Activation of pro-survival or pro-metastasis signaling pathways, such as PI3K/AKT, NF- $\mathrm{kB}$, MAPK and $\beta$-catenin, has been shown to play a role in mediating oncogenic effects in human NSCLC (20-23). To explore the mechanism of barbaloin on the viability and motility of NSCLC cells, the level of AKT, NF- $\kappa B, p 38$ MAPK and $\beta$-catenin and their phosphorylated forms was measured by western blot analysis (Fig. 4A). No difference was observed in the activated (phosphorylated) forms of AKT, NF- $\mathrm{KB}$ and $\beta$-catenin in A549 cells treated with barbaloin in various concentrations, but the level of p-p38MAPK was significantly reduced by barbaloin, especially at 50 and $100 \mu \mathrm{M}$ (Fig. 4B). To verify the inactivating role of barbaloin in $\mathrm{p} 38 \mathrm{MAPK}$ pathway, the expression of downstream genes of p38MAPK (p-Cdc25B and p-Hsp27) was measured by western blot analysis. The result revealed that the level of p-Cdc25B and p-Hsp27 was strongly decreased by barbaloin. Moreover, the subcellular localization of p38 was measured. Cells in control group $(0 \mu \mathrm{M})$ showed accumulated p38 staining in cytoplasm and nucleus. Cells treated with 50 and $100 \mu \mathrm{M}$ barbaloin displayed reduced cytoplasmic and nuclear staining of p38 (Fig. 4C). These results indicated that barbaloin restrained the activation of p38MAPK/Cdc25B/ Hsp27 pathway through inhibition of p38 nucleus translocation.

Barbaloin restrains the growth and hepatic metastases of A549 cells in nude mice. To investigate the effects of barb- 
A

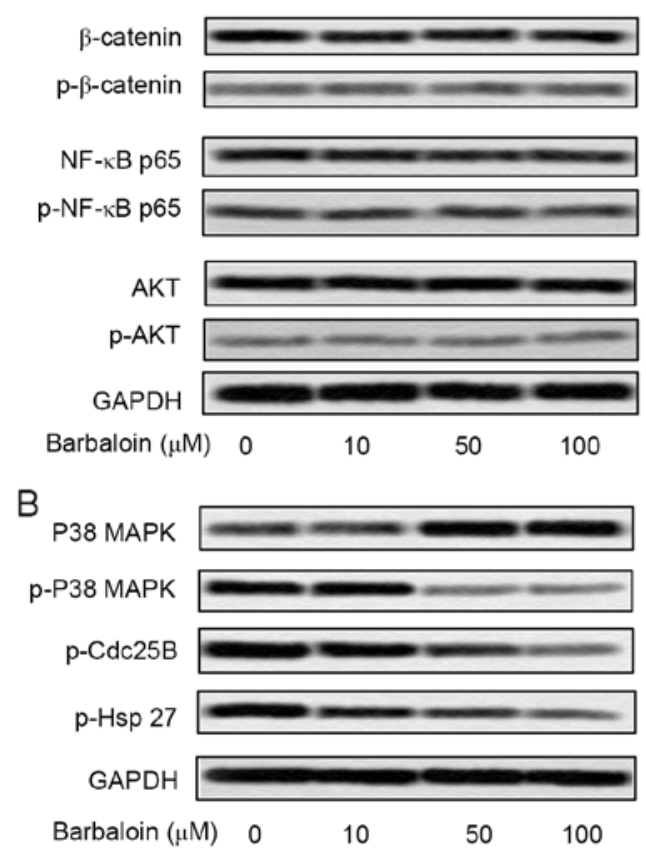

C
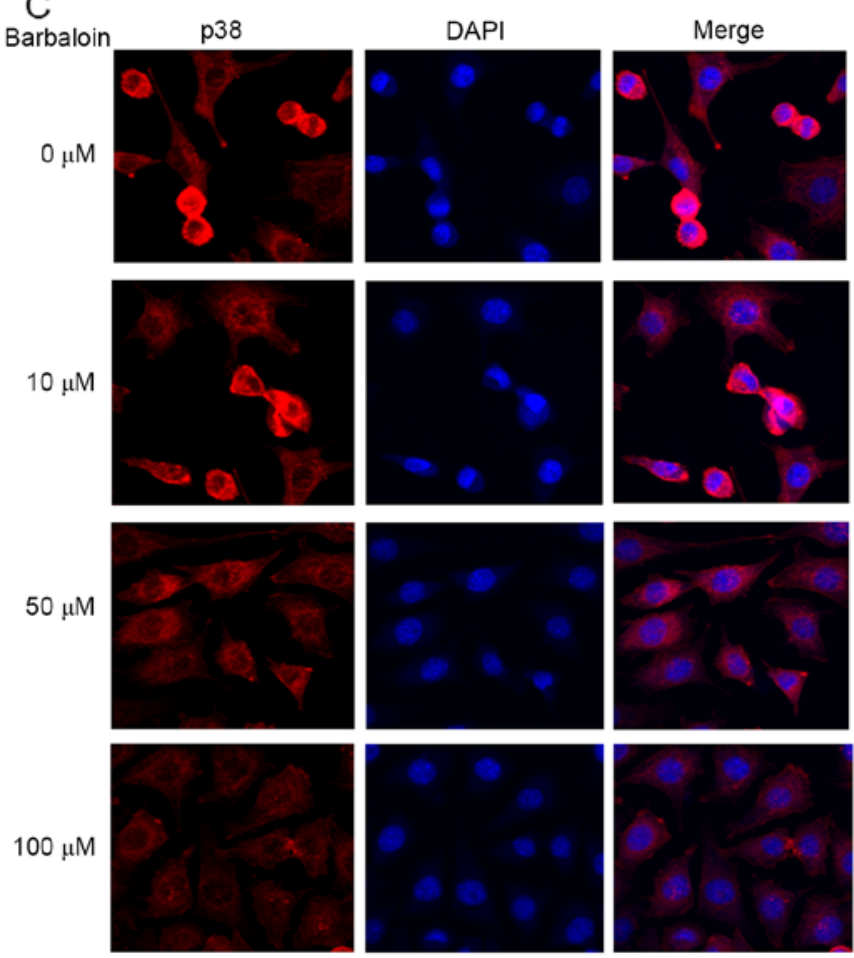

Figure 4. Barbaloin inactivates the P38 MAPK/Cdc25B/Hsp27 pathway. A549 cells were treated with barbaloin at different concentrations (0, 10,50 and

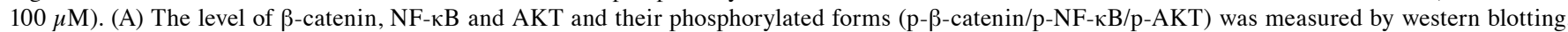
(B) The expression of p38MAPK, p-p38MAPK and its downstream genes (p-Cdc25B/p-Hsp27) was evaluated through western blotting. GAPDH was used as an endogenous reference. (C) Fluorescently-labeled A549 cells were treated with barbaloin, and incubated with corresponding primary and secondary antibodies. After counterstained with DAPI, the subcellular localization of p38 was examined under a fluorescence microscope.

aloin on NSCLC cell migration and invasion in vivo, xenograft mouse model was created by subcutaneous injection of A549 cells pretreated with or without barbaloin $(50 \mu \mathrm{M})$ into SPF nude mice. Barbaloin effectively suppressed tumor formation and tumor volume compared with the control group (Fig. 5A and B). The level of proliferation markers (Ki-67 and PCNA) was also decreased in barbaloin treated mice (Fig. 5C). These results suggested that barbaloin restrained tumor growth of NSCLC in vivo. Besides, the level of tumor metastasisrelated proteins (MMP-9 and VEGF) was also downregulated by barbaloin in NSCLC model mice (Fig. 5D). Fluorescent labeled recombinant A549 cells were found to metastasize to the liver. Fluorescence signal in liver was observed directly through IVIS Spectrum system. We can see that fluorescence signal was significantly reduced by $50 \mu \mathrm{M}$ barbaloin (Fig. 5E). The expression level of p38MAPK (p-Cdc25B and p-Hsp27) was also decreased by $50 \mu \mathrm{M}$ barbaloin (Fig. 5F). These results indicated that barbaloin restrains the growth and hepatic metastases of A549 cells in nude mice and may involve inactivation of the $\mathrm{p} 38 \mathrm{MAPK}$ pathway.

\section{Discussion}

Lung cancer is one of the most malignant cancers in the world (24). Worst still, in China, more and more patients younger than 45 years suffer from lung cancer (25). NSCLC is the most common type of lung cancers (4). At present, chemotherapy combined with surgical resection is the most commonly used method in the treatment of NSCLC. However, the treatment of lung cancer is still not satisfactory with many side-effects and recurrence. Therefore, it is critical to deeply understand the exact molecular mechanisms related to NSCLC development and progression and develop new therapeutic methods for medication and treatment.

In recent years, natural herbal products are applied to the treatment of cancers due to their antitumor activities including apoptosis induction and anti-proliferative activities. For example, phloretin induced apoptosis of NSCLC A549 cells (26). Matrine promoted cell apoptosis in NSCLC by activating p38 (27). These natural compounds effectively restricted the proliferation of cancers with little side-effects. Barbaloin used in the present study is a natural compound extracted from aloe, and has been identified effective in various types of cancer (10-13). A previous research proved that barbaloin significantly inhibited the proliferation, migration and tube formation of HUVECs (human umbilical vein endothelial cells) in vitro by suppressing the activation of VEGF (vascular endothelial growth factor) receptor 2 and STAT3 phosphorylation (10). Similarly, in this study, barbaloin effectively suppressed the cell growth and expression of proliferation markers (Ki-67 and PCNA), especially at higher concentrations $(50$ and $100 \mu \mathrm{M})$. These results indicate that barbaloin inhibits the viability of NSCLC cells.

Accumulated studies have reported the anti-viability effect of barbaloin. Reports have suggested that the combination of aloe with chemotherapy could increase the efficacy in both tumor regression rate and survival time (28). Some research pointed out that Aloe emodin promoted cell cycle arrest and 

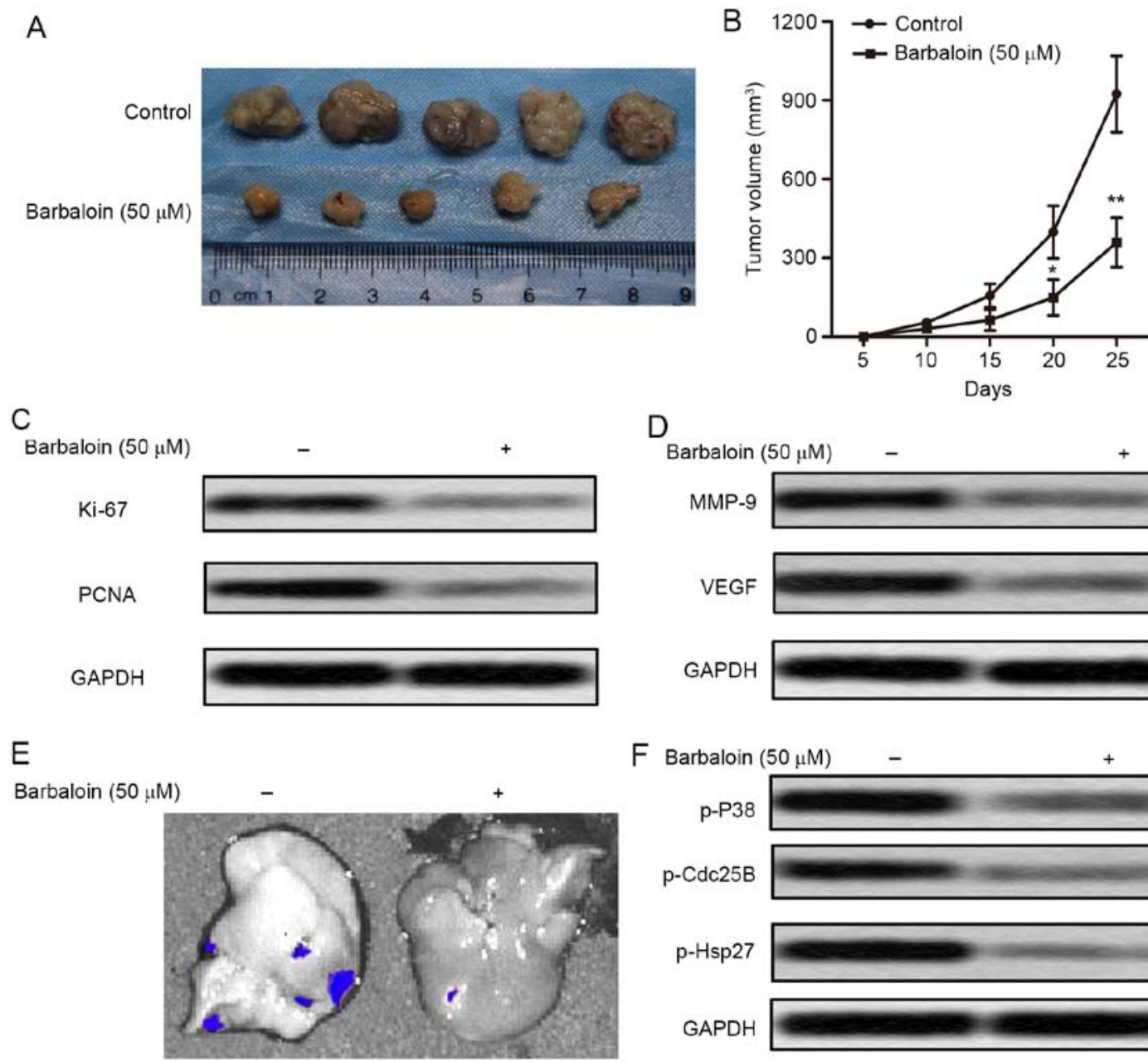

D

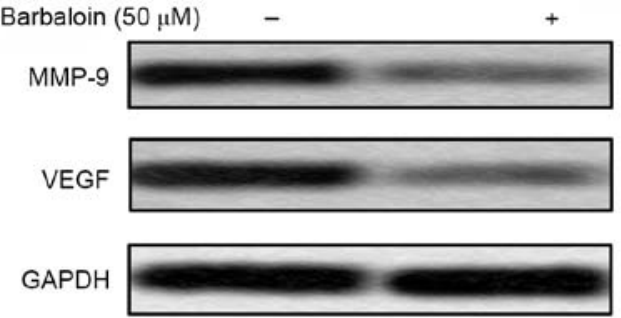

F Barbaloin $(50 \mu \mathrm{M}) \quad-\quad+$

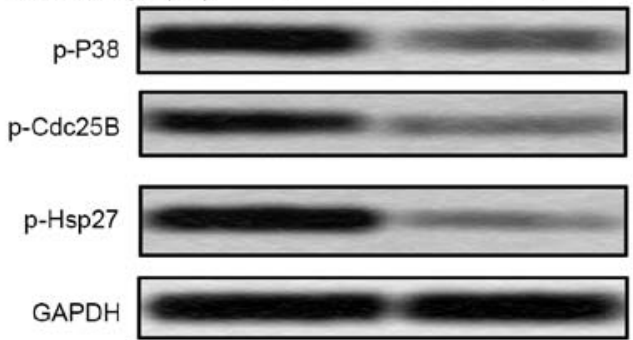

Figure 5. Barbaloin restrains the growth and hepatic metastases of A549 cells in nude mice. Fluorescently-labeled A549 cells were pretreated with or without barbaloin $(50 \mu \mathrm{M})$. NSCLC xenograft mouse model was established by injecting the treated A549 cells into SPF nude mice subcutaneously. (A) The representative images of tumor in each group are presented. (B) Tumor volume was calculated and is shown as means $\pm \mathrm{SD}(\mathrm{n}=5)\left({ }^{*} \mathrm{P}<0.05\right.$, ${ }^{* *} \mathrm{P}<0.01$ vs. control group). (C) The level of proliferation markers (Ki-67 and PCNA) in barbaloin treated mice was detected through western blotting. (D) The expression of tumor metastasisrelated proteins (MMP-9 and VEGF) was evaluated through western blotting. GAPDH was used as an endogenous reference. (E) Livers were removed from mice in two groups. Fluorescence signal in liver was observed directly through IVIS Spectrum system. (F) The expression level of p38MAPK, p-Cdc25B and p-Hsp27 in barbaloin treated mice was detected through western blotting. GAPDH was used as an endogenous reference.

apoptosis in human U87 malignant glioma cells via the mitochondrial membrane potential disruption (29). In this study, the cell viability was largely reduced in A549 cells treated with barbaloin compared with the control group, the inhibition rates were augmented with the increasing concentration of barbaloin. Besides, barbaloin increased the apoptosis rate of A549 cells and induced an accumulation of G2/M phase. Increased expression of apoptosis-related proteins (caspase-3, -8 and -9$)$ and the changed levels of cell cycle checkpoint proteins (p27, p53 and cyclin A) further convinced the antiviability effect of barbaloin in A549 cells. The results above indicated that barbaloin inhibited cell viability of NSCLC via suppressing proliferation, inducing cell apoptosis and G2/M cell cycle arrest.

Barbaloin has also been indicated to possess anti-metastasis effect. According to the research of He et al (14), the invasion and metastasis ability was largely reduced in breast cancer MDA-MB-231 cells treated with aloe emodin. Aloeemodin has also been reported to inhibit the metastasis of HO-8910PM cells by reducing the expression of FAK (focal adhesion kinase) protein and mRNA (15). Similar conclusion was drawn in this study. The invasion and migration ability of A549 cells was largely suppressed under barbaloin treatment. The levels of tumor metastasis-related proteins (MMP-2, MMP-9, MMP-14 and VEGF) were also decreased in A549 cells treated with barbaloin, especially at higher concentrations (50 and $100 \mu \mathrm{M})$. These results suggest the inhibition effect of barbaloin on the motility of NSCLC.

Activation of pro-survival or pro-metastasis signaling pathways has been shown to play a role in mediating oncogenic effects in human NSCLC. Research has proven that aloe-emodin induced apoptosis in human H460 lung nonsmall carcinoma cells by regulating the expression of protein kinase $\mathrm{C}, \mathrm{Bcl}-2$, caspase-3 and p38 protein (17), and others indicated that aloe-emodin induced chondrogenic differentiation of ATDC5 cells via MAP kinases and BMP-2 signaling pathways (30). In this study, the level of classical signaling pathway marker AKT, NF- $\mathrm{B}, \mathrm{p} 38 \mathrm{MAPK}$ and $\beta$-catenin and their phosphorylated forms was measured in barbaloin-treated A549 cells by western blot analysis. The obviously decreased 
level of p-p38MAPK indicated that p38MAPK is inactivated in A549 cells under barbaloin treatment. The decreased expression of p-Cdc25B and p-Hsp27 (downstream genes of p38MAPK) was further verification. In addition, immunofluorescence staining assay showed that barbaloin reduced cytoplasmic and nuclear staining of p38 in A549 cells. The research above indicated that barbaloin restrained the activation of $\mathrm{p} 38 \mathrm{MAPK} / \mathrm{Cdc} 25 \mathrm{~B} / \mathrm{Hsp} 27$ pathway via inhibiting $\mathrm{p} 38$ nucleus translocation.

Aloe also exhibits tumor suppression effects in vivo. Aloeemodin has been reported to suppress the gowth of prostate cancer in an athymic nude mouse model (31). In vivo study showed the positive result of antitumor activity of aloe-emodin in nude mice injected with human HER-2-overexpressing breast cancer cells (32). The antineoplastic properties of aloe-emodin were also observed in highly metastatic B16-F10 melanoma murine cells (33). In this study, barbaloin effectively suppressed tumor formation and tumor volume in the xenograft mouse model. Besides, barbaloin suppressed expression level of proliferation markers (Ki-67 and PCNA) and tumor metastasis-related proteins (MMP-9 and VEGF) in vivo. The fluorescent labeled A549 cells metastasized to liver and barbaloin largely reduced the fluorescence intensity in the liver. The expression level of p38MAPK and downstream genes was also significantly decreased by barbaloin. These results indicated that barbaloin restrains the growth and hepatic metastases of A549 cells in nude mice and may involve inactivation of p38MAPK/Cdc25B/Hsp27 pathway.

In conclusion, the present study found that barbaloin inhibited the cell viability by suppressing cell proliferation, inducing cell apoptosis and G2/M cell cycle arrest in A549 cells. Besides, barbaloin inhibited cell motility by restraining the invasion and migration ability of A549 cells and the level of tumor metastasis-related proteins. Moreover, barbaloin inhibited tumor growth and hepatic metastases of A549 cells in nude mice and this may include restraining the p38MAPK/ $\mathrm{Cdc} 25 \mathrm{~B} / \mathrm{Hsp} 27$ pathway. This study may provide new aspects into the mechanism of NSCLC proliferation and an approach for the treatment of NSCLC.

\section{Acknowledgements}

The present study was funded by the Natural Science Foundation of China (no. 81473775) and the Young Teacher Support Project of Beijing University of Chinese Medicine (no. 2011-JYB22-JS122).

\section{References}

1. Zhang BY, Wang YM, Gong H, Zhao H, Lv XY, Yuan GH and Han SR: Isorhamnetin flavonoid synergistically enhances the anticancer activity and apoptosis induction by cis-platin and carboplatin in non-small cell lung carcinoma (NSCLC). Int J Clin Exp Pathol 8: 25-37, 2015.

2. Verdecchia A, Francisci S, Brenner H, Gatta G, Micheli A, Mangone L and Kunkler I; EUROCARE-4 Working Group: Recent cancer survival in Europe: A 2000-02 period analysis of EUROCARE-4 data. Lancet Oncol 8: 784-796, 2007.

3. D'Arcangelo M and Hirsch FR: Clinical and comparative utility of afatinib in non-small cell lung cancer. Biologics 8: 183-192, 2014.

4. Jemal A, Siegel R, Xu J and Ward E: Cancer statistics, 2010. CA Cancer J Clin 60: 277-300, 2010.
5. Asamura H, Chansky K, Crowley J, Goldstraw P, Rusch VW, Vansteenkiste JF, Watanabe H, Wu YL, Zielinski M, Ball D and Rami-Porta R; International Association for the Study of Lung Cancer Staging and Prognostic Factors Committee, Advisory Board Members, and Participating Institutions: The International Association for the Study of Lung Cancer Lung Cancer Staging Project: Proposals for the Revision of the N Descriptors in the Forthcoming 8th Edition of the TNM Classification for Lung Cancer. J Thorac Oncol 10: 1675-1684, 2015.

6. Avelino CU, Cardoso RM, Aguiar SS and Silva MJ: Assessment of quality of life in patients with advanced non-small cell lung carcinoma treated with a combination of carboplatin and paclitaxel. J Bras Pneumol 41: 133-142, 2015.

7. Waller DA: Neoadjuvant chemotherapy in non small cell lung cancer-the UK experience. Lung Cancer 34 (Suppl 3): S31-S33, 2001.

8. Azim HA Jr and Ganti AK: Treatment options for relapsed small-cell lung cancer. Anticancer Drugs 18: 255-261, 2007.

9. Tabolacci C, Rossi S, Lentini A, Provenzano B, Turcano L, Facchiano $\mathrm{F}$ and Beninati S: Aloin enhances cisplatin antineoplastic activity in B16-F10 melanoma cells by transglutaminase-induced differentiation. Amino Acids 44: 293-300, 2013.

10. Pan Q, Pan H, Lou H, Xu Y and Tian L: Inhibition of the angiogenesis and growth of Aloin in human colorectal cancer in vitro and in vivo. Cancer Cell Int 13: 69, 2013.

11. Esmat AY, Tomasetto C and Rio MC: Cytotoxicity of a natural anthraquinone (Aloin) against human breast cancer cell lines with and without ErbB-2: Topoisomerase IIalpha coamplification. Cancer Biol Ther 5: 97-103, 2006.

12. Esmat AY, El-Gerzawy SM and Rafaat A: DNA ploidy and S phase fraction of breast and ovarian tumor cells treated with a natural anthracycline analog (aloin). Cancer Biol Ther 4: 108-112, 2005.

13. Nićiforović A, Adzić M, Spasić SD and Radojcić MB: Antitumor effects of a natural anthracycline analog (Aloin) involve altered activity of antioxidant enzymes in HeLaS3 cells. Cancer Biol Ther 6: 1200-1205, 2007.

14. He ZH, Huang YQ, Weng SF, Tan YR, He TP, Qin YM and Liang NC: Effect of Aloe emodin on invasion and metastasis of high metastatic breast cancer MDA-MB-231 cells. Zhong Yao Cai 36: 1481-1485, 2013 (In Chinese).

15. He TP, Yan WH, Mo LE and Liang NC: Inhibitory effect of aloeemodin on metastasis potential in HO-8910PM cell line. J Asian Nat Prod Res 10: 383-390, 2008.

16. Lin ML, Lu YC, Chung JG, Wang SG, Lin HT, Kang SE, Tang $\mathrm{CH}$, Ko JL and Chen SS: Down-regulation of MMP-2 through the p38 MAPK-NF-kappaB-dependent pathway by aloe-emodin leads to inhibition of nasopharyngeal carcinoma cell invasion. Mol Carcinog 49: 783-797, 2010.

17. Yeh FT, Wu CH and Lee HZ: Signaling pathway for aloe-emodininduced apoptosis in human H460 lung nonsmall carcinoma cell. Int J Cancer 106: 26-33, 2003.

18. He H, Zheng L, Sun YP, Zhang GW and Yue ZG: Steroidal saponins from Paris polyphylla suppress adhesion, migration and invasion of human lung cancer A549 cells via down-regulating MMP-2 and MMP-9. Asian Pac J Cancer Prev 15: 10911-10916, 2014.

19. Mou H, Zheng Y, Zhao P, Bao H, Fang W and Xu N: Celastrol induces apoptosis in non-small-cell lung cancer A549 cells through activation of mitochondria- and Fas/FasL-mediated pathways. Toxicol In Vitro 25: 1027-1032, 2011.

20. Fei F, Li X, Xu L, Li D, Zhang Z, Guo X, Yang H, Chen Z and Xing J: CD147-CD98hc complex contributes to poor prognosis of non-small cell lung cancer patients through promoting cell proliferation via the PI3K/Akt signaling pathway. Ann Surg Oncol 21: 4359-4368, 2014.

21. Gastonguay A, Berg T, Hauser AD, Schuld N, Lorimer E and Williams CL: The role of Rac1 in the regulation of NF- $\kappa$ B activity, cell proliferation, and cell migration in non-small cell lung carcinoma. Cancer Biol Ther 13: 647-656, 2012.

22. Chien ST, Lin SS, Wang CK, Lee YB, Chen KS, Fong Y and Shih YW: Acacetin inhibits the invasion and migration of human non-small cell lung cancer A549 cells by suppressing the p38 $\alpha$ MAPK signaling pathway. Mol Cell Biochem 350: 135-148, 2011.

23. Miao Y, Wang L, Zhang X, Xu X, Jiang G, Fan C, Liu Y, Lin X, Yu J, Zhang Y, et al: Promoter methylation-mediated silencing of $\beta$-catenin enhances invasiveness of non-small cell lung cancer and predicts adverse prognosis. PLoS One 9: e112258, 2014. 
24. McGuire S: World Cancer Report 2014. Geneva, Switzerland: World Health Organization, International Agency for Research on Cancer, WHO Press, 2015. Adv Nutr 7: 418-419, 2016.

25. Zhang J, Chen SF, Zhen Y, Xiang J, Wu C, Bao P, Luketich J, $\mathrm{Hu} \mathrm{H}$, Zhou X, Zhang J, et al: Multicenter analysis of lung cancer patients younger than 45 years in Shanghai. Cancer 116 3656-3662, 2010.

26. Min J, Huang K, Tang H, Ding X, Qi C, Qin X and Xu Z: Phloretin induces apoptosis of non-small cell lung carcinoma A549 cells via JNK1/2 and p38 MAPK pathways. Oncol Rep 34: 2871-2879, 2015.

27. Tan C, Qian X, Jia R, Wu M and Liang Z: Matrine induction of reactive oxygen species activates p38 leading to caspase-dependent cell apoptosis in non-small cell lung cancer cells. Oncol Rep 30: 2529-2535, 2013.

28. Lissoni P, Rovelli F, Brivio F, Zago R, Colciago M, Messina G, Mora A and Porro G: A randomized study of chemotherapy versus biochemotherapy with chemotherapy plus Aloe arborescens in patients with metastatic cancer. In Vivo 23: 171-175, 2009.
29. Ismail S, Haris K, Abdul Ghani AR, Abdullah JM, Johan MF and Mohamed Yusoff AA: Enhanced induction of cell cycle arrest and apoptosis via the mitochondrial membrane potential disruption in human U87 malignant glioma cells by aloe emodin. J Asian Nat Prod Res 15: 1003-1012, 2013.

30. Yang M, Li L, Heo SM and Soh Y: Aloe-emodin induces chondrogenic differentiation of ATDC5 cells via MAP kinases and BMP-2 signaling pathways. Biomol Ther (Seoul) 24: 395-401, 2016.

31. Liu K, Park C, Li S, Lee KW, Liu H, He L, Soung NK, Ahn JS, Bode AM, Dong Z, et al: Aloe-emodin suppresses prostate cancer by targeting the mTOR complex 2. Carcinogenesis 33: 1406-1411, 2012.

32. Ma JW, Hung CM, Lin YC, Ho CT, Kao JY and Way TD: Aloeemodin inhibits HER-2 expression through the downregulation of Y-box binding protein-1 in HER-2-overexpressing human breast cancer cells. Oncotarget 7: 58915-58930, 2016.

33. Tabolacci C, Lentini A, Mattioli P, Provenzano B, Oliverio S, Carlomosti F and Beninati S: Antitumor properties of aloeemodin and induction of transglutaminase 2 activity in B16-F10 melanoma cells. Life Sci 87: 316-324, 2010. 